\title{
Redistribution of Fluorescently Labeled Tubulin in the Mitotic Apparatus of Sand Dollar Eggs and the Effects of Taxol
}

\author{
Yukihisa Hamaguchi ${ }^{1}$, Masaru Toriyama ${ }^{2}$, Hikoichi Sakai $^{2}$ and \\ Yukio Hiramoto ${ }^{1,3}$ \\ 1Biological Laboratory, Tokyo Institute of Technology, Tokyo 152; ${ }^{2}$ Department \\ of Biophysics and Biochemistry, Faculty of Science, University of Tokyo, \\ Tokyo 113, and ${ }^{3}$ Division of Cell Proliferation, National Institute for Basic \\ Biology, Okazaki 444, Japan
}

\begin{abstract}
Fluorescently labeled tubulin was quickly incorporated into the mitotic apparatus when injected into a live sand dollar egg. After a rectangular area $(1.6 \times 16 \mu \mathrm{m})$ of the mitotic spindle was photobleached at metaphase or anaphase by the irradiation of a laser microbeam, redistribution of fluorescence was almost complete within $30 \mathrm{sec}$. The photobleached area did not change in shape during the redistribution. During the period of redistribution, the bleached area moved slightly toward the near pole at metaphase and anaphase (means: 1.6 and $1.8 \mu \mathrm{m} / \mathrm{min}$, respectively). These results indicate that redistribution was not due to the exchange of tubulin subunits only at the ends of microtubules but to their rapid exchange at sites along the microtubules in the bleached region. Furthermore, treadmilling of tubulin molecules along with the spindle microtubules possibly occurred at the rate of $1.6 \mu \mathrm{m} / \mathrm{min}$ at metaphase.

Birefringence of the mitotic apparatus increased with a large increase in both the number and length of astral rays shortly after taxol was injected. However, the microtubules did not all seem to elongate at the same rate but appeared to become equalized in length. Chromosome movement stopped within $60 \mathrm{sec}$ after the injection. Centrospheres became large and the labeled tubulin already incorporated into the centrospheres was excluded from the enlarged centrospheres. Shortly after the labeled tubulin was injected following the injection of taxol, it accumulated in the peripheral region of the centrospheres, suggesting that microtubules first assembled at this region. Fluorescently labeled tubulin in the mitotic apparatus in the egg after injection of taxol was redistributed much more slowly after photobleaching than in uninjected eggs.
\end{abstract}

Fluorescently labeled tubulin has recently been proved to be useful for the investigation of tubulin dynamics in the mitotic apparatus in living cells $(7,18,20,25)$. Evidence has been presented that labeled tubulin which had been injected into living cells was quickly incorporated into the mitotic apparatus and that photobleaching of the mitotic apparatus was quickly followed by uniform redistribution of fluorescence there. These results suggest that tubulin exchange in microtubules is 
faster than that expected from in vitro experiments (3) and that the exchange occurs not only at the ends of microtubules but also along their entire length.

Taxol, an alkaloid isolated from the western yew, Taxus brevifolia, is known to stabilize microtubules and to enhance assembly of microtubules in vivo as well as in vitro (for a review, see reference 4). It is also utilized to isolate microtubular structures $(23,24)$ and the mitotic apparatus (12).

In this study, we investigated tubulin dynamics in the mitotic apparatus of sand dollar eggs by microinjection of fluorescently labeled tubulin and taxol to observe fluorescence redistribution after a local area in the mitotic apparatus was photobleached.

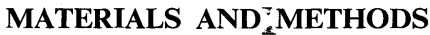

Preparation of fluorescently labeled tubulin. Porcine brain tubulin was labeled fluorescently by three methods:

(a) Microtubule proteins were labeled with 5-(4,6-dichlorotriazin-2-yl) amino fluorescein (DTAF) after Keith et al. (11) and then fluorescently labeled tubulin was separated by phosphocellulose column chromatography as described earlier (7).

(b) Phosphocellulose-purified tubulin was assembled into microtubules at $37^{\circ} \mathrm{C}$, labeled with FITC, and then purified as described earlier (7).

(c) Phosphocellulose-purified tubulin was labeled with DTAF as reported by Soltys and Borisy (22).

Preparation of eggs and microinjection. We obtained gametes of the sand dollar Clypeaster japonicus by the injection of $0.5 \mathrm{M} \mathrm{KCl}$ or sea water containing $1 \mathrm{mM}$ acetylcholine into the coelomic cavity. The fertilization membrane and hyaline layer were removed by treatment of the eggs with $1 \mathrm{M}$ urea for $1 \mathrm{~min}$ shortly after insemination.

We carried out microinjection at $25 \pm 1^{\circ} \mathrm{C}$ as described by Hamaguchi et al. (7). Taxol was dissolved in dimethylsulfoxide (DMSO) at 1 or $2 \mathrm{mM}$ and used for microinjection.

Photomicroscopy and laser photobleaching. Observation and photomicroscopy were carried out using a fluorescence microscope (OPTIPHOT, Nikon, Tokyo) equipped with a UVF40x objective lens (NA 1.30) as reported previously (7). Micrographs were taken directly on $35 \mathrm{~mm}$ film, or they were taken at an exposure of $0.5 \mathrm{sec}$ using a video camera equipped with a silicon-intensified target tube during fluorescence redistribution after laser photobleaching. The time after photobleaching was determined by the clock in the fluorescence micrographs superimposed with a videotimer (VTG-33, For-A, Tokyo).

A laser beam from an argon laser system ( $6 \mathrm{~mW}$ of output power at $488 \mathrm{~nm}$; a GLG 3012 laser head and a GLS 3010 power supply, NEC, Tokyo) and epifluorescence illumination were mixed with a polarization beam splitter which was set between a dichroic mirror and a field diaphragm for epifluorescence. The laser beam $(0.8 \mathrm{~mm}$ in diameter) was limited with a slit $(0.08 \mathrm{~mm}$ in width) made by two pieces of razor blade parallelly set at the image plane of the objective between the beam splitter and the laser system. The specimen was irradiated for $0.54 \mathrm{sec}$ with the laser beam using a shutter set between the slit and the laser system. The laser beam passed through the slit, the beam splitter, an interference filter for excitation, the dichroic mirror, an intermediate lens $(1.25 \mathrm{x})$ and the objective (UVF40x); it then irradiated a rectangular area of $1.6 \mu \mathrm{m} \times 16 \mu \mathrm{m}$ on the specimen. Laser beam irradiation neither perturbed mitosis nor reduced birefringence of the mitotic apparatus. 


\section{RESULTS}

Fluorescently labeled tubulin prepared by method (a) or (b) (see MATERIALS AND MethoDs) was mainly used in the following section 1, and labeled tubulin prepared by method (a) was used in section 2. All three types of labeled tubulin were incorporated quickly into the mitotic apparatus, whose fluorescence was redistributed shortly after photobleaching.

Fluorescence redistribution after photobleaching. When fluorescently labeled tubulin was injected into fertilized eggs at prophase, it was quickly incorporated into the asters near the nucleus and then into the mitotic apparatus formed after the disappearance of the nuclear envelope, as described earlier (7). When the asters or the spindle with labeled tubulin was photobleached by the laser beam at adequate regions at any stage from prophase through telophase, the fluorescence was quickly and uniformly redistributed. Two typical examples of fluorescence redistribution in the mitotic spindle are shown in Fig. 1. In Figs. 1a-e, the long side of the rectangular bleached area was perpendicular to the spindle axis, and in Figs. 1f-j it was parallel
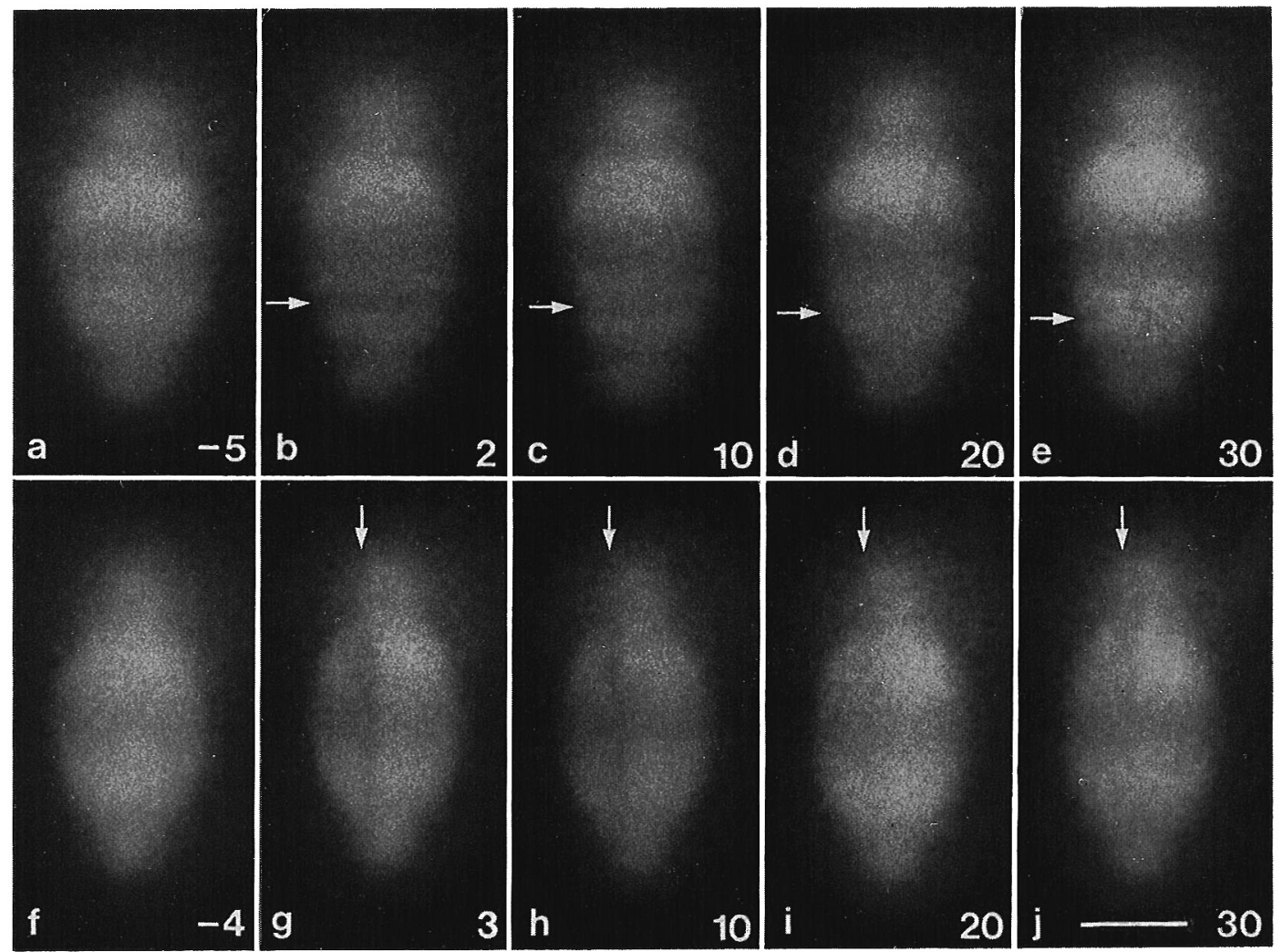

Fig. 1. Two series of fluorescence micrographs of anaphase mitotic apparatuses in which fluorescence was redistributed after photobleaching. Micrographs in the upper and lower rows show the process of the fluorescence redistribution when the photobleached area was perpendicular and parallel to the spindle axis, respectively. The arrow indicates the photobleached area. The numbers in these micrographs are the time $(\mathrm{sec})$ after the end of the laser irradiation. Fluorescently labeled tubulin was injected into sand dollar eggs at prophase. Bar, $10 \mu \mathrm{m} . \times 1,200$. 
to the axis. In these examples, the redistribution was almost complete by $30 \mathrm{sec}$ after photobleaching. The bleached area did not change in shape.

In order to determine whether or not the bleached area moves in the direction of the spindle axis, quantitative measurements of the changes in the distance of the bleached area from the equatorial plane of the spindle (DB) and the distance of the pole of the spindle from the equatorial plane (DP, namely half the spindle length) were made in labeled spindles which had been photobleached perpendicularly (cf. Fig. 1a-e).

The elongation rate of the half spindle and that of the distance from the equator to the bleached area (RP and RB, respectively), which were calculated from DP, DB, and the time after photobleaching using the least-squares method, are plotted in Fig. 2. RP was greatly variable during mitosis; i.e., the spindle did not elongate at metaphase but elongated during anaphase. On the other hand, RB, with mean values of $1.6 \mu \mathrm{m} / \mathrm{min}$ for metaphase spindles and $1.8 \mu \mathrm{m} / \mathrm{min}$ for anaphase spindles, was nearly equal to the rate of half spindle elongation (RP) during early and mid-anaphase $(1.9 \mu \mathrm{m} / \mathrm{min}$; mean value of 5 samples), which means that the bleached area moved $0.8-0.9 \mu \mathrm{m}$ (about half the width of the bleached area) toward the pole during $30 \mathrm{sec}$ of fluorescence redistribution.

Effects taxol microinjection on the mitotic apparatus. When $15 \mathrm{pl}(\sim 2 \%$ of the egg volume) of 1 or $2 \mathrm{mM}$ taxol was injected into fertilized eggs, birefringence of the asters before the nuclear envelope breakdown and that of the mitotic apparatus

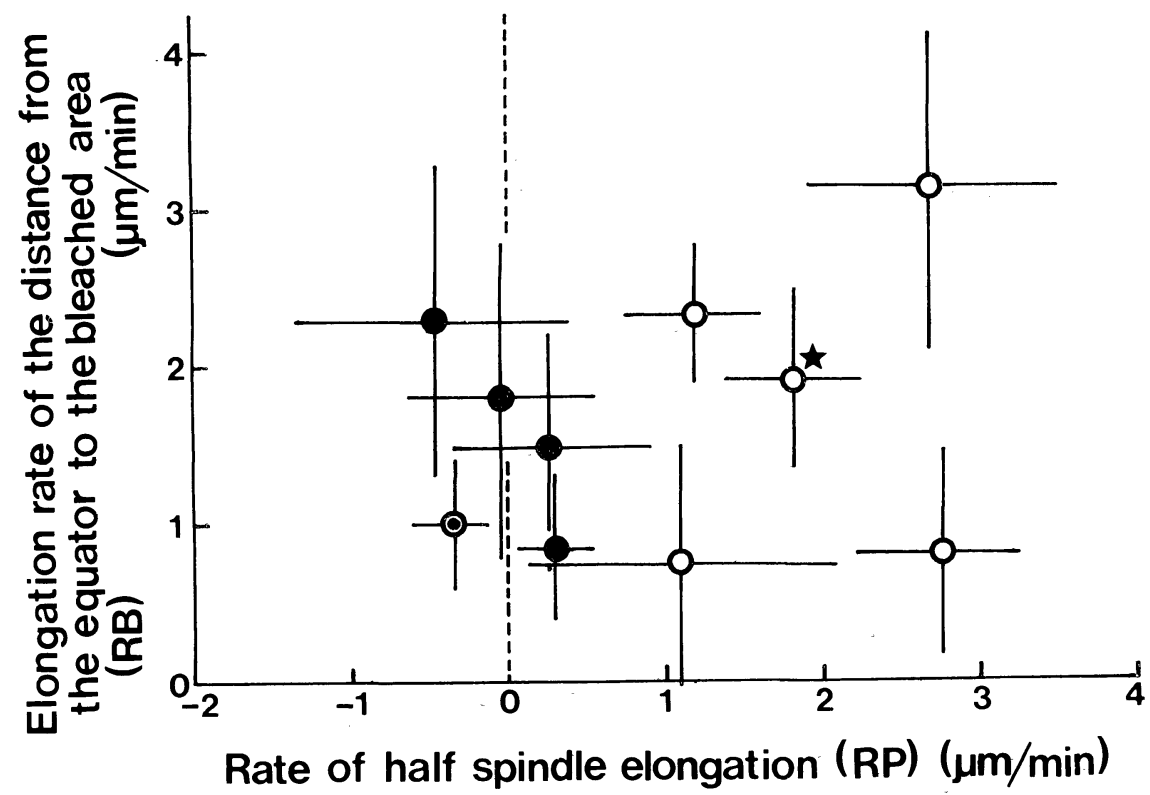

Fig. 2. The rate of half spindle elongation (RP) versus the rate of the elongation of the distance between the center of the bleached area and the equator (RB). Closed, open, and concentric circles represent the values at metaphase, early and mid-anaphase, and late anaphase, respectively. The bars of the crosses represent the standard deviations. The star indicates the datum obtained from the upper row of Fig. 1. The mean rate from 5 examples of the half spindle elongation (RP) at early and mid-anaphase was $1.9 \mu \mathrm{m} / \mathrm{min}$. The mean rate from 4 examples of $\mathrm{RB}$ at metaphase and that from 5 examples of RB at early and mid-anaphase were 1.6 and $1.8 \mu \mathrm{m} / \mathrm{min}$, respectively. 
during mitosis were gradually increased, and astral rays also increased both in number and length (Fig. 3). The astral rays did not appear to elongate uniformly but appeared eventually to become rearranged into uniform lengths. Cytoplasmic granules were excluded from these asters and the diameter of centrospheres increased. Therefore the shape of the asters, including the half spindle, changed to the form of two concentric circles (Fig. 3g, and cf. Fig. 5). Chromosome movement stopped within $60 \mathrm{sec}$ after injection, though later formation of chromosomal vesicles or of the nuclear envelope took place cyclically during division cycles. A cleavage furrow sometimes appeared without completion of cleavage. When $15 \mathrm{pl}$ of DMSO (the solvent for the taxol) was injected alone as a control, neither increase in the birefringence of the mitotic apparatus nor perturbation of mitosis was observed except for infrequent retardation of cleavage.

When first fluorescently labeled tubulin and then taxol were injected into fertilized eggs, the labeled tubulin was greatly accumulated into the mitotic apparatus as well

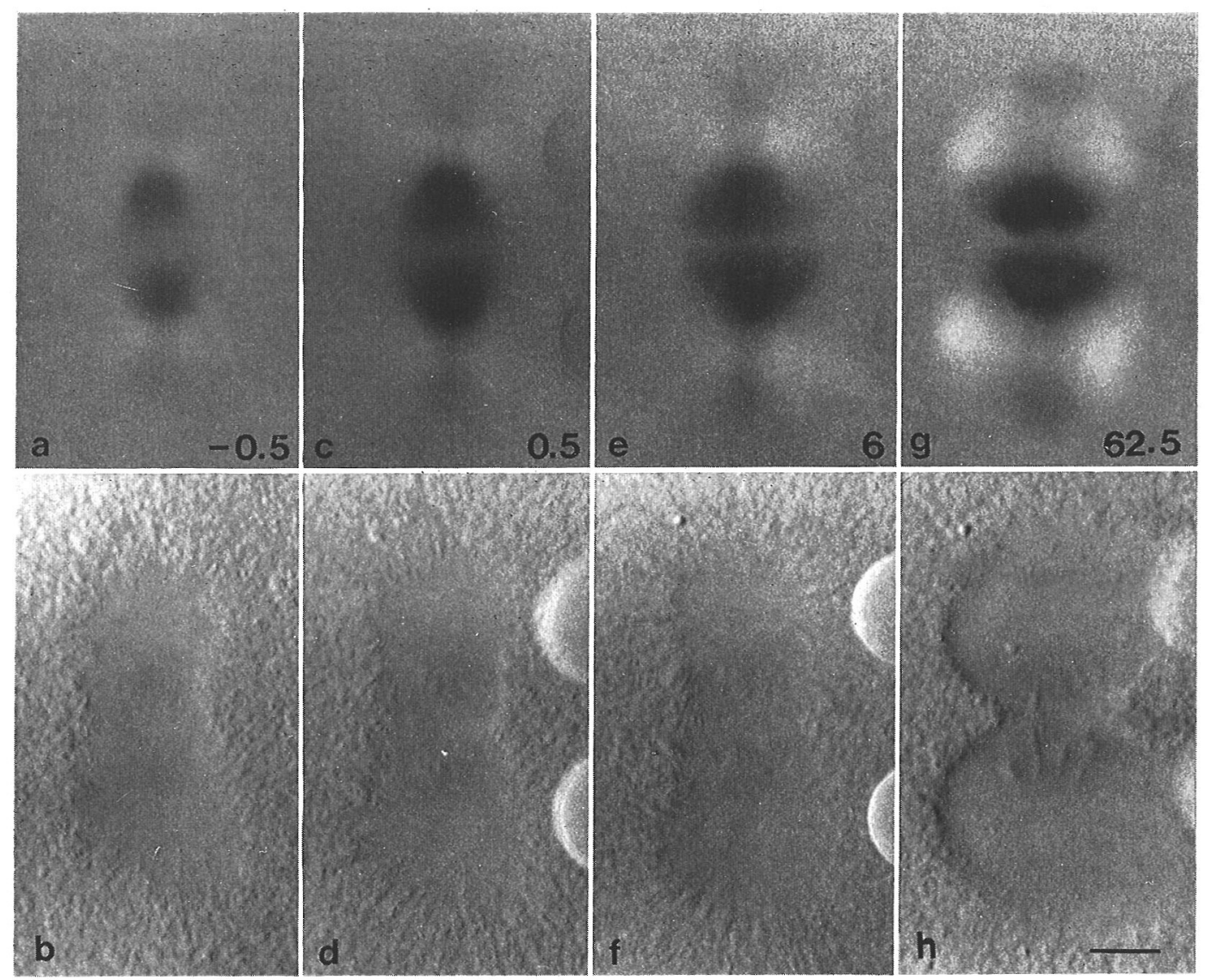

Fig. 3. Effects of taxol on birefringence of the mitotic apparatus and chromosome movement. An egg was injected with $15 \mathrm{pl}$ of $2 \mathrm{mM}$ taxol $66.5 \mathrm{~min}$ after insemination. The numbers in the micrographs are the time ( $\mathrm{min}$ ) after injection of taxol. Polarization micrographs are shown in the upper row with 6.6-nm compensation and the corresponding differential interference micrographs in the lower row. Bar, $10 \mu \mathrm{m}$. $\times 800$. 

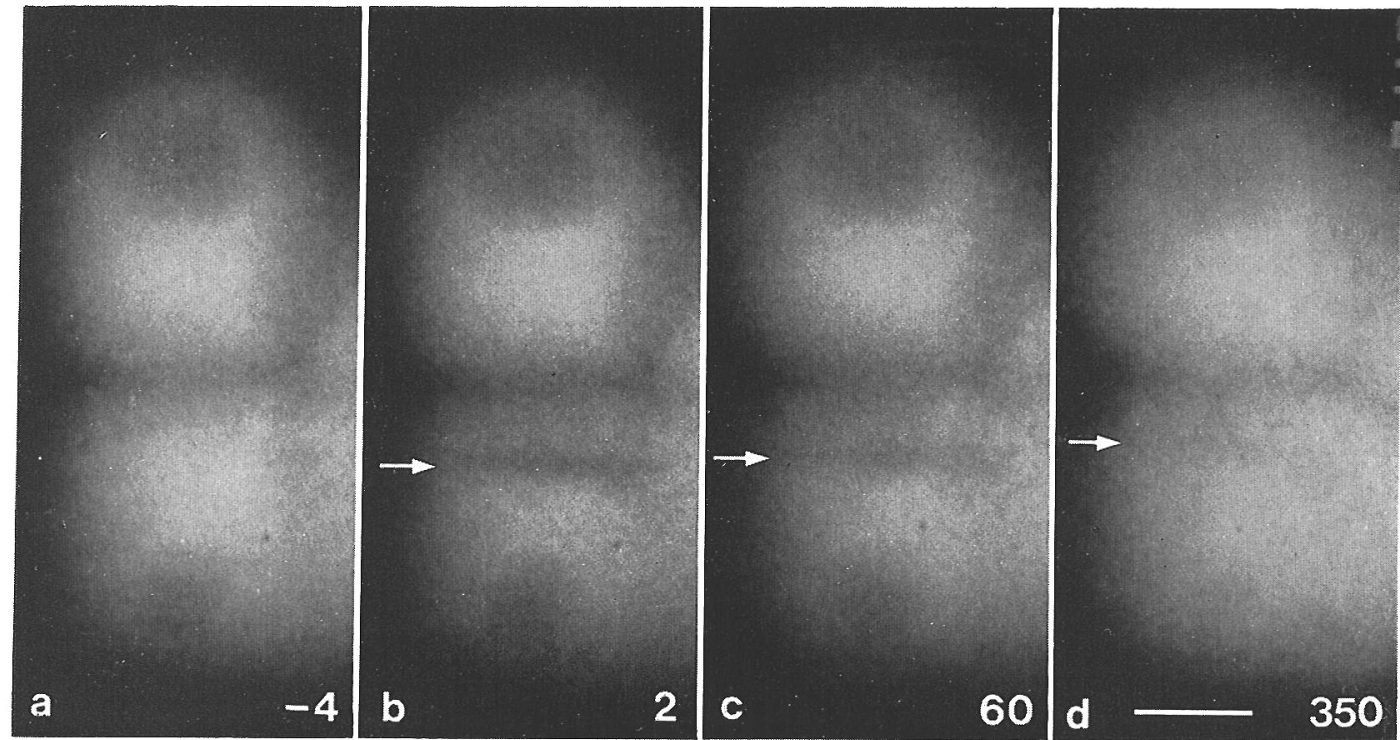

Fig. 4. Fluorescence redistribution in the anaphase mitotic apparatus incorporated with labeled tubulin in the presence of taxol. Fluorescence was redistributed after the photobleaching. The arrow indicates the photobleached area. The numbers in the micrographs are the time (sec) after laser irradiation. Fluorescently labeled tubulin was injected into sand dollar eggs before the nuclear envelope breakdown, followed by injection of $15 \mathrm{pl}$ of $1 \mathrm{mM}$ taxol at anaphase. Bar, $10 \mu \mathrm{m} . \quad \times 1,200$.

as into the region near the injection site. A pronounced feature was that the labeled tubulin was excluded from the enlarged centrospheres (Fig. 4a). The accumulation of tubulin near the injection site might not be due to DMSO but to a high concentration of taxol there. Taxol may precipitate near the injection site before it uniformly diffuses in the egg cytoplasm because taxol does not dissolve aqueous solution at the concentration of $1 \mathrm{mM}$.

The spindle region was photobleached a few min after taxol injection (Fig. 4b) and then the fluorescence redistribution was examined. Even after $5 \mathrm{~min}$ or more, the photobleached area was clearly observable. Therefore, in the presence of taxol, tubulin exchange in the mitotic apparatus was much slower than in its absence. The position of the photobleached area did not change in the spindle, but in some cases the area appeared to move slightly toward the equator (Fig. 4).

Shortly after the egg was injected with taxol and then fluorescently labeled tubulin, the labeled tubulin has accumulated in the peripheral circular region of the enlarged centrospheres (Fig. 5a), and then fluorescence of the asters became intense and rather uniform (Fig. 5c), which suggests that microtubule assembly first occurred at the peripheral region of the centrospheres shortly after injection. In the cells injected with fluorescently labeled tubulin alone, the fluorescence was at first quickly incorporated into the astral centers (see also reference 7).

\section{DISCUSSION}

When the mitotic apparatus which had incorporated labeled tubulin was photo- 


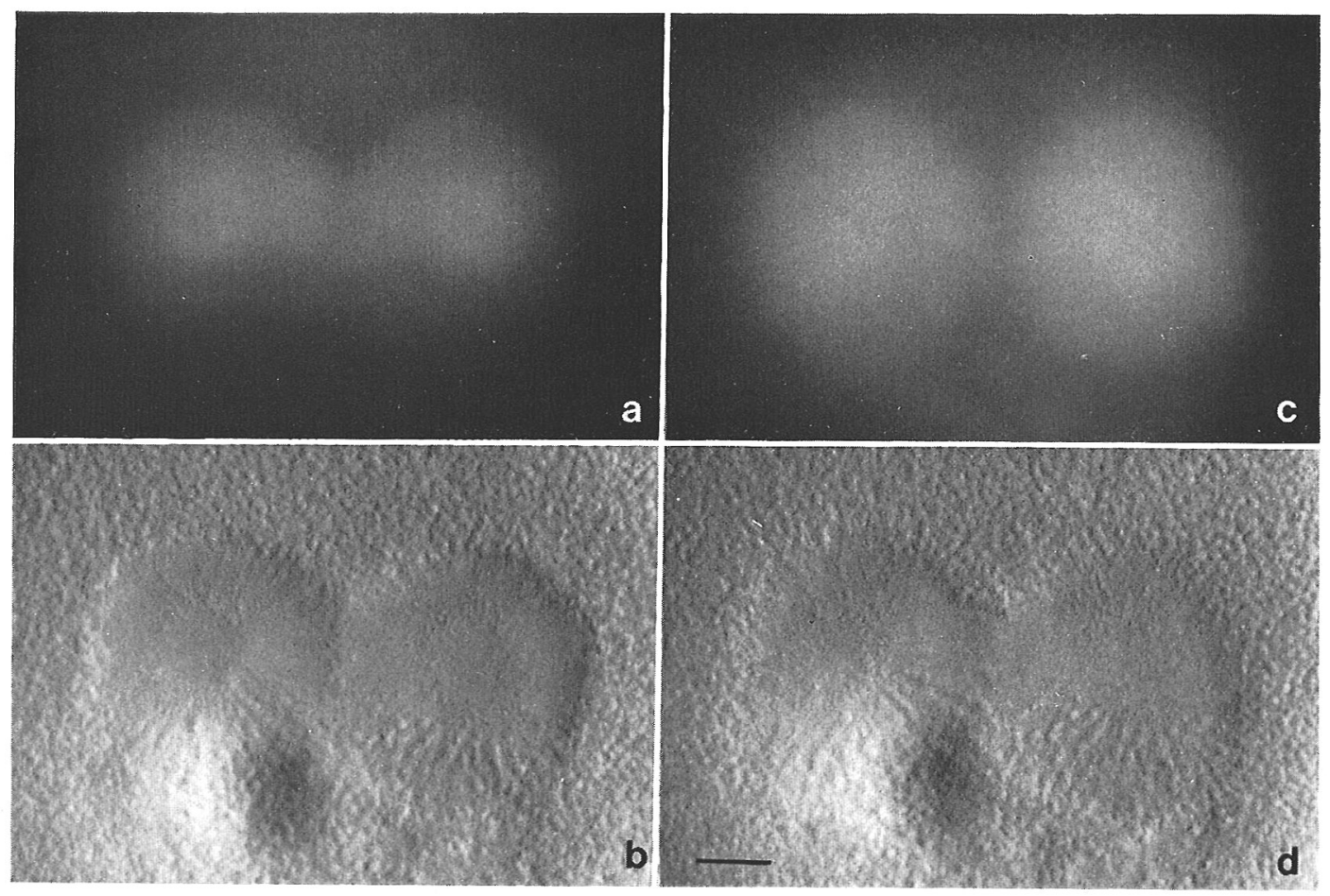

Fig. 5. Incorporation of fluorescently labeled tubulin into the mitotic asters preloaded with taxol. An egg was injected with $15 \mathrm{pl}$ of $1 \mathrm{mM}$ taxol shortly after the nuclear envelope breakdown (47.5 min after insemination) and then with fluorescently labeled tubulin 9 min later. Fluorescence micrographs (a and c) and the corresponding differential interference micrographs (b and d) were taken $1 \mathrm{~min} 55 \mathrm{sec}(\mathrm{a}$ and $\mathrm{b}$ ) and $8 \mathrm{~min} 30 \mathrm{sec}(\mathrm{c}$ and d) after injection of the labeled tubulin. Bar, $10 \mu \mathrm{m} . \quad \times 800$.

bleached by a laser microbeam, fluorescence redistribution occurred within 30 sec. The rate of fluorescence redistribution was consistent with that of incorporation of fluorescently labeled tubulin into the mitotic apparatus after injection (7). The fluorescence recovery was not due to recovery of fluorescence activity of the fluorochrome, fluorescein, but was due to redistribution of fluorescently labeled tubulin in place of the labeled but bleached tubulin. Because the photobleached area did not move as far as the pole along the spindle axis during redistribution and because the redistribution occurred within the same period irrespective of the direction of the longer sides of the bleached rectangle (perpendicular or parallel to the axis), the redistribution may not be due to treadmilling of tubulin subunits in microtubules but to the exchange of tubulin subunits along the microtubules. Alternatively, the rapid redistribution of fluorescence might be explained by rapid asynchronous assembly and disassembly of spindle microtubules, as a phenomenon similar to that described by Mitchison and Kirschner $(13,14)$, if almost all of the preexistent microtubules were disassembled and an equal amount of microtubules were assembled within $30 \mathrm{sec}$. However, this would not be likely because of the large pool size of tubulin in the sea urchin egg (16). 
The present results were repeatedly confirmed using all three types of labeled tubulin and two species of labeling probes (FITC and DTAF). Therefore, our results with the sand dollar agree with the previous results in other living cells with respect to the quick exchange of tubulin $(18,20,25)$. The redistribution in sand dollar eggs was, however, faster than that in cultured cells (25).

Fluorescence redistribution occurred so fast that it was not easy to determine accurately the positions of the poles in the micrographs. Thus the standard deviations on the rates in Fig. 2 are large. Nevertheless, we detected during the short period for fluorescence redistribution that the photobleached area keeps moving toward the pole at a rate of $\sim 1.6 \mu \mathrm{m} / \mathrm{min}$ for metaphase spindles and $\sim 1.8 \mu \mathrm{m} / \mathrm{min}$ for anaphase ones. These values were close to that of the half spindle elongation, $\sim 1.9 \mu \mathrm{m} / \mathrm{min}$, at early and mid-anaphase. This movement was observed even for the metaphase spindle, where no change occurs in the spindle length. This observation might be explained by assuming that the movement is caused only by treadmilling of tubulin subunits in spindle microtubules at metaphase. Tubulin may enter the kinetochore microtubule end (plus end) and exit from the polar end (minus end). The value of $1.6 \mu \mathrm{m} / \mathrm{min}$ for the movement of the bleached area was reasonable as compared with the rate of treadmilling expected from in vitro experiments $[\sim 1 \mu \mathrm{m} /$ min, (3)]. At anaphase, growth of interzonal microtubules may occur, which in turn results in elongation of the spindle. Chromosomes move toward the poles at a velocity of $\sim 1 \mu \mathrm{m} / \mathrm{min}$ in the egg of this species of sand dollar [anaphase A, (9)], which may accelerate this poleward movement of the bleached area, provided that the bundles of kinetochore microtubules move toward the pole at the same velocity as chromosomes do. However, the movement of the bleached area was a little slower than that expected for the anaphase spindle [ $\sim 3 \mu \mathrm{m} / \mathrm{min},(9)]$, the reason for which remains to be solved.

That the bleached area moved toward the pole in this study contradicts the results reported by Wadsworth and Salmon (24), in which the bleached area was absolutely at a standstill during redistribution. Although the inconsistency cannot be solved at present, the present results are rather consistent with those previously reported by Forer (6), who observed that the area of reduced birefringence formed by ultraviolet microbeam irradiation in the spindle of crane fly spermatocytes moved poleward at the same velocity as anaphase chromosomes. Similar poleward movements of endogenous markers in the spindle were reported by Allen et al. (1) in Haemanthus endosperm and by Hiramoto and Izutsu (10) in grasshopper spermatocytes. Hiramoto observed similar movement in sand dollar eggs (unpublished data).

Microinjection of taxol has the merit of quickly affecting the microtubular structures in big cells such as the sand dollar eggs in the present study and amphibian oocytes (8), but has the demerit of uneven concentration of taxol in the egg cytoplasm because of its low solubility in aqueous medium, which results in the promotion of microtubule assembly at the injection site independent of microtubule-organizing centers. The taxol injection induced a birefringence increase in the mitotic apparatus, which was caused by microtubule assembly depending on microtubule-organizing centers and suppression of chromosome movement as reported previously in cells cultured in a taxol-containing medium $(2,4,15,19)$. Moreover, according to the experiment of fluorescence redistribution after photobleaching, taxol reduced the exchangeability of tubulin in microtubules. Reduced exchange of tubulin subunits was also reported in an in vitro experiment (see reference 4). In the present experiment, 
a remarkable effect of taxol on the mitotic apparatus was that the lengths of microtubules in the asters including the half spindle became equalized. The experiments involving injection of taxol and tubulin suggest that microtubule-organizing granular materials (see reference 5) dispersed from the center of the asters to the periphery of the enlarged centrospheres and that microtubules disappeared from the centrospheres. Enlarged monopolar aster and centrosphere were also observed during fertilization by means of taxol injection (our unpublished data) and incubation in a taxolcontaining medium (21). The effects of taxol on the mitotic structures quite resemble those of hexyleneglycol on the mitotic apparatus with respect to changes in distribution of microtubule-organizing granules and in morphology of microtubular cytoskeletons for asters and spindle $(5,17)$.

Acknowledgment. We wish to thank the Misaki Marine Biological Station for supplying sand dollars and Dr. M. Suffness at the National Cancer Institute (Maryland, USA) for the generous gift of taxol. The authors' thanks are also due to Dr. I. Mabuchi for the use of a laser system.

This work was supported by Grants-in-Aid for Scientific Research form the Japanese Ministry of Education, Science and Culture awarded to Y. Hamaguchi (59740359), Y. Hiramoto (5848002), and H. Sakai (60065005).

\section{REFERENCES}

1. Allen, R.D., A. Bajer and J. LaFountain. Poleward migration of particles or states in spindle fiber filaments during mitosis in Haemanthus. J. Cell Biol. 43, 4a, 1969

2. CANDE, W.Z. A permeabilized cell model for studying cytokinesis using mammalian tissue culture cells. J. Cell Biol. 87, 326-335, 1980

3. COTE, R.H. and G.G. BorisY. Head-to-tail polymerization of microtubules in vitro. J. Mol. Biol. 150, 577-602, 1981

4. De Brabander, M., G. Geuens, R. Nuydens, R. Willebrords, F. Aerts and J. De Mey. Microtubule dynamics during the cell cycle: The effects of taxol and nocodazole on the microtubule system of PtK2 cells at different stages of the mitotic cycle. in International Review of Cytology, Vol. 101, ed. Bourne, G.H., J.F. Danielli and K.W. Jeon, Academic Press, New York, pp. 215-274, 1986

5. Endo, S., M. ToriYama and H. Sakai. The mitotic apparatus with unusually many microtubules from sea urchin eggs treated by hexyleneglycol. Develop. Growth Differ. 25, 307-314, 1983

6. FoRer, A. Local reduction of spindle fiber birefringence in living Nephrotoma suturalis (Loew) spermatocytes induced by ultraviolet microbeam irradiation. J. Cell Biol. 25, 95-117, 1965

7. Hamaguchi, Y., M. Toriyama, H. Sakai and Y. Hiramoto. Distribution of fluorescently labeled tubulin injected into sand dollar eggs from fertilization through cleavage. J. Cell Biol. 100, 1262-1272, 1985

8. Heidemann, S.R. and P.T. Gallas. The effect of taxol on living eggs of Xenopus laevis. Dev. Biol. 80, 489-494, 1980

9. Hiramoto, Y., Y. Hamaguchi, Y. Shoji, T.E. Schroeder, S. Shimoda and S. Nakamura. Quantitative studies on the polarization optical properties of living cells. II. The role of microtubules in birefringence of the spindle of the sea urchin egg. J. Cell Biol. 89, 121-130, 1981

10. Нiramoto, Y. and K. Izutsu. Poleward movement of "markers" existing in mitotic spindles of grasshopper spermatocytes. Cell Struct. Funct. 2, 257-259, 1977

11. Keith, C.H., J.R. Feramisco and M. Shelanski. Direct visualization of fluorescein-labeled microtubules in vitro and in microinjected fibroblast. J. Cell Biol. 88, 234-240, 1981

12. KuriYAma, R., G. KerYer and G.G. Borisy. The mitotic spindle of Chinese hamster ovary cells isolated in taxol-containing medium. J. Cell Sci. 66, 265-275, 1984 
13. Mitchison, T. and M. Kirschner. Microtubule assembly nucleated by isolated centrosomes. Nature, 312, 232-237, 1984

14. Mitchison, T. and M. Kirschner. Dynamic instability of microtubule growth. Nature, 312, 237-242, 1984

15. Mole-Bajer, J. and A.S. Bajer. Action of taxol on mitosis: Modification of microtubule arrangements and function of the mitotic spindle in Haemanthus endosperm. J. Cell Biol. 96, 527-540, 1983

16. Pfeffer, T.A., C.F. Asnes and L. Wilson. Properties of tubulin in unfertilized sea urchin eggs. Quantitation and characterization by the colchicine-binding reaction. J. Cell Biol. 69, 599-607, 1976

17. Rebhun, L.I. and N. Sawada. Augmentation and dispersion of the in vivo mitotic apparatus of living marine eggs. Protoplasma, 68, 1-22, 1969

18. Salmon, E.D., R.J. Leslie, W.M. Saxton, M.L. Karow and J.R. McIntosh. Spindle microtubule dynamics in sea urchin embryos: Analysis using a fluorescein-labeled tubulin and measurements of fluorescence redistribution after laser photobleaching. J. Cell Biol. 99, 21652174,1984

19. Sato, H., A. Ковау AShi and T.J. Iтон. The mitotic arresters: Molecular probes of spindle assembly. in Cell Motility: Mechanism and regulation, ed. Ishikawa, H., S. Hatano and H. Sato. Univ. of Tokyo Press, Tokyo, pp. 357-370, 1986

20. Saxton, W.M., D.L. Stemple, R.J. Leslie, E.D. Salmon, M. Zavortink and J.R. McIntosh. Tubulin dynamics in cultured mammalian cells. J. Cell Biol. 99, 2175-2186, 1984

21. Schatten, G., H. Schatten, T.H. Bestor, and R. Balczon. Taxol inhibits the nuclear movements during fertilization and induces asters in unfertilized sea urchin eggs. J. Cell Biol. 94, 455-465, 1982

22. Soltys, B.J. and G.G. Borisy. Polymerization of tubulin in vivo: Direct evidence for assembly onto microtubule ends and from centrosomes. J. Cell Biol. 100, 1682-1689, 1985

23. Vale, R.D., B.J. Schnapp, T. Mitchison, E. Steuer, T.S. Reese and M.P. Sheetz. Different axoplasmic proteins generate movement in opposite directions along microtubules in vitro. Cell 43, 623-632, 1985

24. Vallee, R.B. and G.S. Bloom. Isolation of sea urchin egg microtubules with taxol and identification of mitotic spindle microtubule-associated proteins with monoclonal antibodies. Proc. Natl. Acad. Sci. U.S.A. 80, 6259-6263, 1983

25. Wadsworth, P. and E.D. Salmon. Analysis of the treadmilling model during metaphase of mitosis using fluorescence redistribution after photobleaching. J. Cell Biol. 102, 1032-1038, 1986

(Received for publication, September 5, 1986) 